\title{
A dor irruptiva na doença oncológica avançada*
}

\section{Irruptive pain in advanced cancer}

Ângela Sofia Lopes Simões ${ }^{1}$

* Recebido do Serviço de Cirurgia Mulheres (ULSCB) Hospital Amato Lusitano. Castelo Branco, Portugal.

- Estudo elaborado durante a I Pós-Graduação em Cuidados Paliativos da Escola Superior de Saúde Dr. Lopes Dias.

\section{RESUMO}

JUSTIFICATIVA E OBJETIVOS: A doença oncológi$\mathrm{ca}$, atendendo à sua natureza, percurso e tratamento assume-se numa dimensão especial pelo enorme impacto no indivíduo nos níveis físico, psicológico, espiritual, social, na família e comunidade. O sintoma mais temido pelos pacientes e familiares é indubitavelmente a dor. Apesar de nos últimos anos os avanços na área do tratamento da dor terem sido enormes ainda existem lacunas para um tratamento global, como é o caso da dor irruptiva. O objetivo deste estudo foi sistematizar conceitos e definições de dor associados à doença oncológica, entender o mecanismo do aparecimento da dor irruptiva e conhecer as melhores formas para o seu alívio.

CONTEÚDO: Através da revisão bibliográfica encontrou-se as principais barreiras ao tratamento correto deste tipo de dor e as formas mais adequadas, na atualidade, para o seu controle.

CONCLUSÃO: A dor irruptiva é de aparição espontânea, idiopática, não relacionada com uma pauta analgésica determinada, nem a funções ou movimentos corporais.

Descritores: Assistência paliativa, Dor, Neoplasias, Terapêutica.

1. Enfermeira Especialista em Enfermagem Médico-Cirúrgica do Serviço de Cirurgia Mulheres (ULSCB) Hospital Amato Lusitano; Pós-Graduanda em Cuidados Paliativos; Mestre em Tratamento de Suporte e Cuidados Paliativos no Doente Oncológico. Castelo Branco, Portugal.

Endereço para correspondência:

Ângela Sofia Lopes Simões

Av. de Espanha lote 36, $6^{\circ}$ Esquerdo 6000-078

Castelo Branco, Portugal

Telemóvel: 351967080994

E-mail: angela.simoes@gmail.com

\section{SUMMARY}

BACKGROUND AND OBJECTIVES: Cancer, due to its nature, evolution and treatment, is given a special dimension for the huge impact on patients in physical, psychological, spiritual and social levels, in addition to affecting families and the community. Most feared symptom by patients and relatives is undoubtedly pain. Although in recent years there have been major advances in the treatment of pain, there are still gaps for a global treatment, such as irruptive pain. This study aimed at systematizing concepts and definitions of cancer-related pain, at understanding irruptive pain onset mechanism and the best ways to relieve it.

CONTENTS: By reviewing the literature we have found the major barriers for adequately treating this pain and the most adequate ways to currently control it.

CONCLUSION: Irruptive pain is spontaneous, idiopathic, not related to an established analgesic regimen or to body functions or movements.

Keywords: Neoplasias, Pain, Palliative care, Therapy.

\section{INTRODUÇÃO}

Imaginem ter uma dor incompreendida, incapacitante e constante Os pacientes oncológicos não precisam de imaginar, sentem-na! E as famílias vivem-na! A dor é o sintoma que causa mais angústia nos pacientes e familiares, e os pacientes assumem-na como prioritária no seu tratamento, porque influencia o controle de outros sintomas como a insônia, a depressão, a ansiedade, a anorexia, entre outros ${ }^{1}$. Os receios mais frequentes dos pacientes oncológicos são o medo de serem abandonados e a dor. Sem o alívio da dor, a sobrevivência é tida como impossível. Se preocupar com a dor é urgente, porque esta realidade é agora! 
Em 1985 registraram-se aproximadamente cinco milhões de mortes devido a doença oncológica. Estima-se que em 2015 esse número suba para os nove milhões. Setenta a $80 \%$ destes pacientes experimentarão dor moderada a intensa e muitos morrerão com dor não controlada, apesar das evidências mostrarem que em $80 \%$ dos pacientes é possível obter um controle completo com analgésicos orais, quer isolados quer combinados com coadjuvantes ${ }^{2}$. Além disso, estima-se que $20 \%$ das síndromes dolorosas intensas não responderam à terapia instituída baseada na escada analgésica da Organização Mundial de Saúde (OMS), e foram considerados como síndromes dolorosas rebeldes, de difícil controle ou tolerantes à terapêutica com opioides ${ }^{3}$.

Atualmente dispõe-se de formas eficazes de controle da dor, como utilização de analgésicos seguros, incorporação de técnicas inovadoras de anestesia, o uso de grande quantidade de dispositivos para administração de fármacos, protocolos de atuação, criação de unidades específicas para o tratamento da dor. Todas vieram permitir uma melhor e mais adequada analgesia. No entanto, dos vários milhões de pessoas em todo o mundo que sofrem de algum tipo de dor, uma grande percentagem é tratada de forma insuficiente, desadequada ou não recebe qualquer tipo de tratamento por diversos e complexos motivos. As principais razões para esta situação catastrófica são a inexperiência, a falta de conhecimento dos princípios simples e manuseio dos analgésicos efetivos e em muitos países a indisponibilidade de analgésicos opioides fortes ${ }^{2}$. Grande parte dos profissionais de saúde não sabe o que significa "dor" quando falam dela ${ }^{4}$. Em estudo, concluem que atualmente, nos hospitais ainda se trata a dor segundo o seu processo evolutivo e não segundo as particularidades do paciente. Referiu também que a alta prevalência que encontrou se deve à não aplicação das diretrizes da escada analgésica da OMS, já que os analgésicos eram administrados apenas quando o paciente solicitava. A via de administração de eleição era a oral seguida da via venosa e segundo a opinião dos profissionais a via subcutânea era menos eficaz que a muscular considerada analgésica por si só ${ }^{5}$.

Nos últimos anos, muito se tem evoluído em termos de tratamento da dor, em que o estabelecimento de diretrizes e a fundamentação da escada analgésica de OMS em 1980 veio dar um contribuição fundamental. Mas mesmo com a dor basal controlada, um número bastante elevado de pacientes refere dor intermitente e intensa. A dor episódica constitui um fenômeno heterogêneo e frequente nas situações de doença oncológica avançada, diminuindo a qualidade de vida dos pacientes. Até agora, a falta de meios terapêuticos suficientemente eficazes, fez com que os profissionais de saúde desvalorizassem este tema. Atualmente, já existem estudos suficientes que permitem um conhecimento aprofundado do tema para providenciar um cuidado adequado ${ }^{6}$.

Ao consultar autores que muito contribuíram para o estudo da fisiopatologia e tratamento da dor irruptiva, constatou-se de imediato a falta de uniformização em relação aos termos aplicados como sinônimos, sem o serem. Um estudo recente sobre as características da dor oncológica levado a cabo pela Task Force on Cancer Pain of the International Association of the Study of Pain (IASP), encontrou grandes diferenças no diagnóstico e tratamento da dor irruptiva nos diferentes países estudados, através de discussões com vários especialistas de todas as partes do mundo. O termo 'breakthrough pain' é unicamente anglo-saxônico. O termo "episódico" ou "transitório" é o mais referenciado em países como a França, Alemanha, Itália e Espanha. Além das diferenças em termos de nomenclatura, alguns médicos consideram que flutuações numa dor basal devem-se exclusivamente a uma terapia farmacológica inadequada. Na Inglaterra, frequentemente é usado o termo irruptivo e dor de fim de dose de forma indiferenciada. Médicos tunisianos defenderam que exacerbações da dor faziam parte da trajetória normal da doença oncológica, que deveria ser tratada com progressivos aumentos da dose dos analgésicos opioides. A IASP Task Force considera que em muitas partes do mundo o fenômeno da dor irruptiva não está sendo tratado como um síndrome per si que merece um cuidadoso diagnóstico e tratamento específico ${ }^{7}$.

$\mathrm{Na}$ dor episódica podem-se englobar três tipos de dor, que em comum têm o fato de serem transitórias sobre uma dor basal já controlada com fármacos opioides. Diferencia-as em: dor incidental, dor de fim de dose e dor irruptiva ${ }^{6}$. A dor incidental surge de forma esperada após um estímulo voluntário, involuntário ou emocional, muito frequente nos casos de metastização óssea. A radioterapia paliativa pode ter um importante papel no alívio deste tipo de dor quando as lesões dolorosas estão bem localizadas e não são universais. Outra linha de intervenção é o uso de Estrôncio-89 como coadjuvante da radioterapia externa, um radiofármaco capaz de se fixar nas metástases blásticas e promover a analgesia, estratégia especialmente indicada nos casos de múltiplas metástases e distribuídas de forma universal ${ }^{6,8}$. Tem-se também disponível, a administração de inibidores da reabsorção óssea, como os bifosfatos, eficazes no alívio da dor e na prevenção de fraturas a médio e longo prazo. Utiliza-se especialmente nas metástases líticas próprias do câncer da mama e do mieloma 
múltiplo ${ }^{6,9}$. Existem intervenções mais complicadas mas imprescindíveis como a cirurgia descompressiva, no caso de compressão medular e a cirurgia ortopédica no caso de fratura patológica. $\mathrm{Na}$ dor neuropática, apesar de não ser puramente etiológica, deve considerar-se a eficácia dos bloqueios nervosos e dos antidepressivos triciclicos eficazes na dor neuropática lancinante ${ }^{9-11}$. A dor de fim de dose surge sempre antes da hora de administração do fármaco basal eleito e significa necessariamente um desajuste entre a dose do fármaco instituído e a intensidade de dor existente. “... tratamento da dor de fim de dose, é simples: aumentar a dose de opioide até cobrir as necessidades do paciente"

A dor irruptiva é de aparição espontânea, idiopática, não relacionada com uma pauta analgésica determinada, nem a funções ou movimentos corporais e que corresponderá ao Breakthrough pain dos estudos anglo-saxônicos ${ }^{3,6,12,13}$. "Se atendermos ao seu padrão temporal, a dor irruptiva é uma dor de dificil controle"3.

O objetivo deste estudo foi sistematizar conceitos e definições de dor associados à doença oncológica, entender o mecanismo do aparecimento da dor irruptiva e conhecer as melhores formas para o seu alívio.

\section{PREVALÊNCIA}

Os estudos sobre dor irruptiva são ainda escassos, mas demonstram a gravidade da situação e as repercussões nefastas no paciente e seus familiares. Em 1984 foi publicada a monografia "Cancer Pain" pelo Ministério da Saúde Canadense que retoma as ideias deixadas pelo Expert Committee da OMS em 1980, que indicava aos médicos a necessidade de administração de analgésicos opioides "pelo relógio" para controle da dor oncológica. Identificar e tratar a dor irruptiva, era nesta época, um elemento secundário ${ }^{7}$. Um dos primeiros estudos publicados, de referência obrigatória, foi realizado por Portenoy e Hagen em 1990, citado por Nabal e Madrid, onde definiram pela primeira vez a dor irruptiva como um tipo de dor de difícil controle que surgia de forma transitória em $64 \%$ dos pacientes com dor basal controlada ${ }^{6}$. Em outro estudo realizado por Fine e Bush em 1998, citado por Gonçalves, conclui-se que $86 \%$ dos 22 pacientes estudados apresentavam dor irruptiva de grande intensidade, com predomínio nos estados avançados da doença oncológica e durante o período diurno ${ }^{14}$. Portenoy, Payne e Jacobsen ${ }^{12}$, encontraram dor irruptiva em 51,2\% dos 164 pacientes estudados e em $50 \%$ deles não se encontrou nenhum fator desencadeante. No estudo de Nabal e col. ${ }^{3}$ dos 46 pacientes estudados $60 \%$ apresentaram dor contínua e simultanea- mente dor irruptiva, com uma frequência de 3,22 episódios por dia. A intensidade média da dor basal foi medida com a escala analógica visual, onde a classificaram com 29,2/100 e a intensidade média da dor irruptiva em $57,5 / 100$. Em 2000, a literatura sobre dor irruptiva em pacientes oncológicos tornou-se substancial, especialmente nos Estados Unidos da América. Muitas destas novas investigações surgiram devido ao desenvolvimento e teste de novos opioides analgésicos e formas de administração. Simultaneamente o estudo da dor irruptiva ganha adeptos, por se tratar de um desafio em termos de controle da dor?.

\section{FISIOPATOLOGIA E PERFIL DE TRATAMENTO}

A elevada incidência da dor irruptiva em pacientes oncológicos, como demonstram os estudos referidos afeta ainda mais o controle da dor. A frequente aparição de uma dor inesperada, de grande intensidade e sem um mecanismo que a desencadeie provoca a deterioração do estado do paciente e da própria família. A sua fisiopatologia é marcada por diversos itens confirmados nos vários estudos realizados: dor aguda e transitória; em pacientes com dor basal tratada com opioides potentes; a maioria das vezes idiopática ${ }^{3,6}$. Portenoy, Payne e Jacobsen ${ }^{12}$ confirmam estes dados e acrescentam outros de fundamental valor: o número médio de episódios de dor irruptiva é 6 , a dor atinge a sua intensidade máxima após 3 minutos do seu início. Concluem também que a intensidade da dor irruptiva e a frequência dos episódios influenciava a percepção do paciente da sua dor basal, anteriormente controlada. Os pacientes com dor irruptiva apresentavam maior ansiedade e maiores limitações físicas e sociais, o que a transforma num fenômeno prevalente e heterogêneo.

Sendo esta dor de tão difícil controle, o seu tratamento deveria direcionar-se para uma avaliação exaustiva e a aplicação de técnicas farmacológicas e não farmacológicas eficazes. Na avaliação é importante recolher o máximo de dados sobre: topologia, duração, intensidade, existência de fatores desencadeantes, previsibilidade do seu aparecimento, tipologia e etiologia da dor ${ }^{14}$. Com a finalidade de alcançar o tratamento mais idôneo possível é necessário recolher informações em relação à frequência de episódios de dor irruptiva, a temporalidade em relação aos intervalos de administração da dose basal, a localização da dor irruptiva e da basal e a eficácia do seu tratamento, quer da dor irruptiva quer da basa ${ }^{15}$. A fisiopatologia da dor irruptiva é normalmente idêntica à da dor oncológica de base, assim, segundo os tecidos lesionados e a descrição da dor pode ser nociceptiva visceral, nociceptiva somática ou neuropática ${ }^{14}$. 
A estratégia terapêutica para a dor irruptiva normalmente envolve, numa primeira linha um aumentar gradual da dose do opioide prescrito em calendário ou diminuir o intervalo entre as doses para despiste de dor intensa por final de dose. Até agora, a forma mais rápida de obter efeito analgésico é através da administração por via venosa de bolus, que normalmente demora 2 a 5 minutos para a metadona e 15 a 30 minutos para a morfina e hidromorfina. Se disponível, a analgesia controlada pelo paciente (ACP) poderá desempenhar um efeito importante porque permite ao paciente administrar rapidamente doses de resgate em casos de episódios de dor intensa?. " $E$ dificil titular analgésicos opioides para a dor irruptiva, porque, por um lado a dose de opioides que mantêm confortável o paciente durante o dia torna-se ineficaz perante a dor irruptiva, por outro lado, a manutenção da dose de opioide que consegue aliviar a dor irruptiva, durante todo o dia, poderia sedar gravemente o paciente", podendo ser necessárias várias tentativas de titulação de dose até se encontrar a dose mais eficaz ${ }^{16}$.

Como a dor irruptiva envolve intensidade de dor intensa, de início súbito e que se prolonga por um curto período é importante encontrar um opioide que consiga responder a todas estas necessidades. Adequado à intensidade, de efeito terapêutico de inicio rápido e com curto período de ação. Deverá ser usado, sempre que possível, o mesmo opioide instituído para a dor basal. "É menos dispendioso, mais simples e mais fácil de identificar possíveis efeitos secundários, especialmente quando se lida com pacientes polimedicados"16.

Tenta-se encontrar um opioide que apresente maior rapidez de ação ao nível da mucosa oral, com farmacocinética semelhante às fórmulas parenterais e com um farmacodinâmica previsível ${ }^{14}$. A via oral é a mais conveniente e preconizada pela escada analgésica da OMS e a morfina é normalmente o analgésico opioide de primeira escolha? ${ }^{7}$. Deve-se introduzir morfina de ação rápida, numa percentagem de $5 \%$ a $15 \%$ da dose total de morfina. O limite inferior deste intervalo é utilizado em pacientes fragilizados ou que apresentam dor irruptiva moderadamente intensa. O limite superior é utilizado quando se prevê uma dor irruptiva grave e se o risco não for demasiado grande, tendo em conta a idade do paciente e a presença de insuficiência de órgão grave que predisponham à toxicidade opioide ${ }^{12}$. A dose exata de morfina de ação rápida será a dose que alivie a dor sem efeitos secundários graves, por isso é necessária uma individual e cuidadosa titulação ${ }^{7}$. No entanto, a morfina de ação rápida pode demorar entre 30 a 45 minutos até se tornar efetiva, devido à baixa biodisponibilidade oral, relacionada com a intensa biotransformação decorrente da primeira passagem pelo figado ${ }^{15}$. Preparações retais podem conseguir uma analgesia a partir dos 10 minutos. Apesar da administração de comprimidos de morfina não estar ainda autorizada por esta via, estudos empíricos comprovam a sua eficácia ${ }^{1,17}$.

Nos últimos anos foi desenvolvido um fármaco que promete revolucionar o controle da dor irruptiva com o mínimo de efeitos secundários. O OTFC (oral transmucose fentanyl citrate) obtem uma analgesia eficaz porque combina as vantagens da fisiologia da mucosa oral com as físico-químicas próprias do citrato de fentanil. A mucosa oral tem características que a tornam na via de administração de eleição para tratamentos analgésicos rápidos e eficazes: grande área de superfície, temperatura uniforme, alta permeabilidade, boa vascularização, o que permite a rápida absorção de fármacos. A morfina por ser hidrofílica é lentamente absorvida pela mucosa oral, o que leva a analgesias tardias. Pelo contrário, o citrato de fentanil é muito lipofílico o que implica rápidas absorções ao nível transcelular da mucosa oral e uma rápida extensão e distribuição ao sistema nervoso central conduzindo a quase imediatas analgesias ${ }^{18}$. Está indicado em pacientes já controlados com opioides fortes (o equivalente a pelo menos $60 \mathrm{mg} /$ dia de morfina oral) e que conseguem tolerar os efeitos secundários ${ }^{19,20}$. Estudos realizados sobre a farmacocinética do OTFC, concluíram que $25 \%$ do fármaco é imediatamente disponibilizado pela mucosa oral, possibilitando rápida ação analgésica. Dos $75 \%$ restantes, $25 \%$ escapa à eliminação hepática e intestinal de primeira passagem e torna-se sistemicamente disponível e os restantes $50 \%$ não são absorvidos, obtendo assim uma biodisponibilidade de $50 \%$ com um efeito médio de 6 horas, muito semelhante ao efeito da morfina por via venosa ${ }^{15,19}$. Ensaios clínicos, citados por Gracia, concluem também, que o OTFC é o único fármaco que provou ser eficaz e seguro no tratamento da dor irruptiva ${ }^{15}$. O estudo onde se comparou a eficácia do OTCF e a morfina de ação rápida, em 134 pacientes tratados com opioides fortes, concluiu que o OTCF é significantemente mais eficaz que a morfina de ação rápida no tratamento da dor irruptiva e os principais efeitos secundários descritos foram sonolência, náuseas e obstipação $^{13,19,20}$.

Deve-se orientar o paciente a tomar o analgésico de ação rápida imediatamente após o início da dor irruptiva, já que aguardar algum tempo pode fazer com que o seu controle seja mais difícil ${ }^{14}$. "É muito importante explicar ao paciente os beneficios das doses de resgate. Detecta-se uma infrautilização das doses extras relacionadas com a falta de informação" ${ }^{\prime 20}$. O OTFC escapa à regra dos 5\% a $15 \%$ da dose basal de opioide instituída para a dor basal ${ }^{19}$. A 
dose inicial deve ser de $200 \mu \mathrm{g}$, aumentando gradualmente conforme necessário ao longo do intervalo de doses disponíveis $(200,400,600,800,1200$ e $1600 \mu \mathrm{g})$. Se a analgesia adequada não for obtida em 15 minutos após o paciente ter acabado de usar uma única dose, pode ser consumida uma segunda unidade com a mesma dose. Não devem ser utilizados mais do que 2 unidades para tratar uma crise individual de dor irruptiva. Os pacientes deverão ser vigiados cuidadosamente durante o período de titulação até ser atingida a dose que proporciona analgesia adequada com efeitos secundários aceitáveis, utilizando uma única dose de OTFC por cada crise de dor. Se o paciente experimentar mais de 4 episódios de dor irruptiva por dia, ao longo de um período superior a 4 dias consecutivos, a dose de opioide de ação prolongada prescrito para a dor basal deve ser reavaliada ${ }^{18,20}$.

Deve-se orientar os pacientes a guardar convenientemente este fármaco, longe do alcance de crianças e pessoas que não consumam analgésicos opioides, já que a dose de OTFC é potencialmente fatal nestes casos ${ }^{19}$.

A prescrição do OTFC deve ser criteriosa, como para qualquer opioide, e as precauções durante a sua administração são semelhantes às de outros analgésicos opioides agonistas puros ${ }^{15}$. Está contraindicado em pacientes com depressão respiratória grave ou situações graves de obstrução pulmonar e em pacientes medicados com inibidores da monoamino-oxidase (IMAO) ou nas duas semanas após cessar o uso de IMAO. Deve ser administrado com precaução em pacientes com hipotensão, hipovolemia ou insuficiência hepática e renal ${ }^{12,20}$. Deve-se dar especial atenção aos pacientes que apresentem xerostomia ou mucosite porque a eficácia do fármaco pode estar diminuída. Nos casos de xerostomia deve-se ensinar o paciente a hidratar a boca com bochechos de água antes da administração do OTFC, nos casos de mucosite extensa, além da eficácia do fármaco ficar seriamente comprometida a sua utilização pode ser extremamente dolorosa para o paciente.

Medidas não farmacológicas, como a mudança de decúbitos, reabilitação e controle de sintomas associados podem aliviar a dor irruptiva ${ }^{7}$. Pode-se também associar aos opioides de ação rápida técnicas não farmacológicas comportamentais que poderão ajudar no controle da ansiedade provocada pelos episódios de dor irruptiva, como a visualização criativa, relaxamento e distração. A visualização consegue uma libertação mental efetiva devido à abstração que induz através da imaginação do paciente; o relaxamento consegue um efeito benéfico ao nível da diminuição da tensão muscular e como indutor do sono; a distração permite ao paciente concentrar-se numa ativida- de agradável, especialmente importante quando aguarda o início do efeito terapêutico dos analgésicos ${ }^{21}$. Pode-se também testar a eficácia de técnicas de estimulação cutânea, como o TENS, a aplicação de calor e frio, a acupuntura e massagens de pressão, que em alguns tipos de dor poderão conseguir efetiva diminuição ou bloqueio da percepção de dor. As técnicas de biofeedback e hipnose podem ser úteis por fornecer as formas de controlar períodos de ansiedade e assim evitar a exacerbação da percepção da dor ${ }^{21}$.

\section{CONCLUSÃO}

Uma dor intensa que não é aliviada acarreta consequências físicas, psicológicas e sociais adversas. O paciente tem direito a um tratamento adequado à sua dor e que se respeite a sua avaliação individual. Além disso, o não tratamento da dor por "ignorância", por "medo das reações adversas", ou por negligência leva à violação grave de princípios bioéticos perfeitamente estabelecidos. Quando apesar de existirem diversas técnicas de alívio da dor estudadas e aceitas por organismos mundiais, não são utilizadas, viola-se o princípio da beneficência e da não maleficência. Quando existe uma grande percentagem de pacientes que não têm acesso aos meios de controle da dor, viola-se o princípio da justiça. Quando se incute nos pacientes o medo no uso de opioides fortes devido ao enraizamento de mitos ridículos e infundados, viola-se o princípio da autonomia, ao privá-lo de uma opção consciente e segura.

Quando se consegue observar nos pacientes, pessoas com realidades individuais, em vez de casos clínicos, talvez se consiga perceber que tem-se que ir mais além da medicação analgésica. Porque os medicamentos podem tratar a dor mas não curam todas as dores. A pior de todas é a dor da alma, a dor dos afetos ou a dor da falta dos afetos, e essa se trata com disponibilidade, empatia, carinho, humor e profissionalismo.

\section{REFERÊNCIAS}

1. Twycross R. Introducing palliative care. $4^{\text {th }}$ ed. Oxford: Radcliffe Medical Press; 2003. p.208

2. Hanks GW. Cancer pain and the importance of its control. Londres. Anticancer Drugs 1995;6(Supp1 3):14-7.

3. Nabal M, Naudí C, Ramírez M, et al. Prevalencia del dolor episódico y continuo en cuidados paliativos. Rev Soc Esp Dolor 2001;8 (4):265-8.

4. Pessini L. Humanização da dor e sofrimento huma- 
no no contexto hospitalar. Rev Bioética 2002;10(2):51-72 5. Padrol A, Pérez-Esquiva M, Olona M. Estúdio de la prevalência del dolor crónico en pacientes hospitalizados. Rev Soc Esp Dolor 2001;8 (8):555-61.

6. Nabal M, Madrid F. Dolor episódico: definición, etiología y fisiopatología. Rev Soc Esp Dolor 2002;9 (2):88-93.

7. Colleau S. The significance of breakthrough pain in cancer. WHO, Cancer Pain Release 1999;12(4):1-8.

8. López González JL, Molina Villaverde R. Protocolo de empleo paliativo de la radioterapia. Indicaciones y limitaciones. Medicine 2001;8(57):3069-70

9. Márquez Martínez E, Canudas M, Martínez R, et al. Fármacos coadyuvantes en el tratamiento del dolor oncológico. Dolor: investigación, Clínica \& Terapêutica 2009;24(2):89-95

10. Caraceni A, Martini C, Zecca E, et al. Breakthrough pain characteristics and syndromes in patients with cancer pain. An international survey. Palliat Med 2004;18(3):177-83.

11. Oliveira AS, Torres Hde P. Anesthetic blocks to treat cancer pain. Rev Bras Anestesiol 2003;53(5):654-62.

12. Portenoy RK, Payne D, Jacobsen P. Breakthrough pain: characteristics and impact in patients with cancer pain. Pain 1999;81(1-2):129-34.

13. Zeppetella G. Fentanyl buccal tablet for cancer breakthrough pain. Future Prescriber 2008;9(1):5-9.

14. Gonçalves JA. The importance of a word: how to translate 'breakthrough'. Palliat Med 2008;22(2):195.
15. Vara F, Ortega E, Centeno C, et al. Citrato de fentanilo oral transmucosa: una nueva fórmula de liberación para el tratamiento del dolor irruptivo. Medicina Paliativa 2002;9(1):6-9

16. Bruera E. Management of breakthrough pain due to cancer - The Simmonds article reviewed. Oncology 1999;13 (8):1110-3.

17. Gomez Sancho M, Ojeda M, Garcia Rodrigues E, et al. Medicina Paliativa en la Universidad. La experiencia de Las Palmas de Gran Canaria. Medicina Paliativa 2006;4(13):192-6.

18. Muriel C, Gárcia-Castano M, Yusta G et al. Citrato de fentanilo transmucosa oral (OTFC): revision. Rev Soc Esp Dolor 2000;7 (5):319-26.

19. Payne R, Coluzzi P, Hart L, et al. Long-term safety of oral transmucosal fentanyl citrate for breakthrough cancer pain. J Pain Symptom Manage 2001;22(1):575-83.

20. Coluzzi PH, Schwartzberg L, Conroy JD, et al. Breakthrough cancer pain: a randomized trial comparing oral transmucosal fentanyl citrate (OTFC) and morphine sulfate immediate release (MSIR). Pain 2001;91(1-2):123-30.

21. Gili F, Gracia A. Breakthrough pain: a specific entity that needs an appropiate treatment. Medicina Paliativa 2001;2(8):109-12.

Apresentado em 13 de janeiro de 2011.

Aceito para publicação em 12 de maio de 2011. 